\title{
A Short Analysis of the Nature of Reading
}

\author{
Feng Liu
}

School of Foreign Languages, Qingdao University of Science and Technology, Qingdao, China 266061

\author{
E-mail: liufeng7079@163.com
}

This paper is partial achievement of the teaching research and reform project of Qingdao University of Science and Technology "The Use of Campus Network Based Computerized Adaptive Testing in College English Test"

\begin{abstract}
This paper gives a short analysis of the nature of reading. Though it is generally believed that reading involves perceiving the written form of language, the term reading has not been clearly defined up to date. It is possible to see reading as a process, or to examine the product of that process. Three reading models, namely Bottom-up Model, Top-down Model and Interactive Model, are discussed in this paper.
\end{abstract}

Keywords: Reading, Reading models, Bottom-up Model, Top-down Model, Interactive Model

\section{Introduction}

Reading is such a pervasive activity that we all know what reading is, yet the term "reading" has not been clearly defined up to date. Reading usually means dealing with language messages in written or printed form, it involves processing language messages, hence knowledge of language. Widdowson (1979) defines reading as " the process of getting linguistic information via print". By talking about "getting information", Widdowson appears to imply that this is a fairly one-way process from writer or text to reader, although Widdowson would not want to appear to be implying this, since he has been an important advocate of the view that the reader interprets and contributes to incoming messages. The problem is present in an ambiguity in Widdowson's definition. One would say that "linguistic information" is restricted to information about, say, syntax, morphology and lexis. Actually the information can be of any kind that is encoded in language. So reading is "the process of receiving and interpreting information encoded in language form via the medium of print"'(Urquhart \& Weir, 1998).

Clearly, reading involves perceiving the written form of language. Do readers relate the printed form of language to the spoken form? Or, does word recognition go from visual input to sound and then to meaning? If so, then once that first transformation has taken place, reading is the same sort of activity as listening, and the only specific aspect of reading that testers need to concern with is the process of transformation from print to speech. This is often referred to as the phonemic or phonological route in literature. The other argument, put forward by theorists like Smith (1971) is that readers proceed directly to meaning, and do not go via sound. This is known as the direct route, i.e. word recognition goes straight from the visual input to meaning without recourse to sound.

Rayner \& Pollatsek (1989), after careful investigation, conclude that direct visual access is important and that sound encoding plays some part.

There is a growing consensus in the recent cognitive psychology research literature that all reading requires what is called "early phonological activation", in other words, readers typically identify the sound of words as part of the process of identifying their meaning. Electromyographic recording also shows that subvocalisation is a normal part of silent reading. However, research is unclear on whether the phonological identification proceeds independently of the use of semantic and other uses, or whether it is sequential, proceeding in stages, i.e. sound is recognized first, then meaning.

Juel (1991, cited in Urquhart \& Weir, 1998) cites evidence that early attainment of word recognition or decoding skill is a very accurate predictor of later reading comprehension in first language (L1) children. Recent accounts of fluent reading tend to emphasize that it is rapid, and increasing importance has been attributed to automatic word recognition. Beck (1981, cited in Urquhart \& Weir, 1998) even argues that automatic word recognition is more important to fluent processing of text than content clues. It is now generally accepted that automatic word recognition is a distinctive feature of good reading.

Research into the development of critical reading skills or abilities draws upon the study of reading and thinking. Indeed, many aspects of reading represent problem-solving, the problem-solving strategies are useful for the resolution of many difficulties. Thorndike, as early as 1917, characterized reading as reasoning. By this he meant that many of the strategies by which readers resolve matters of meaning approximate to a logical process of deduction and inference, and that good readers are those who can think clearly. Those persuaded of the value of teaching critical reading will very probably feel a need to test such abilities. However, test constructors should 
know to what extent they wish to assess reading ability, and to what extent they wish to distinguish this from other cognitive abilities. It is at least intuitively possible to make a distinction between the ability to read and the ability to think critically. However, the attempt to gain a picture of somebody's reading abilities uncontaminated by other cognitive variables, i.e. to keep reading separate from reasoning is fraught with difficulties (Alderson, 2000).

Despite the impression that may have been created in this section so far, reading is not an isolated activity that takes place in some vacuum, it is not merely a cognitive operation of meaning extraction. There is an increasing tendency to see reading as only one of a number of literacy practices. According to Bernhardt (1991), "taking a cognitive perspective means examining the reading process as an intrapersonal problem-solving task that takes place within the brain's knowledge structures". As a social process, Bloome \& Greene (1984, cited in Urquhart \& Weir, 1998) argue that "reading is used to establish, structure, and maintain social relationships between and among peoples". Reading, as a social activity, is related to particular contexts, as Wallace (1992) puts it: "As readers we are frequently addressed in our social roles rather than our personal and individual ones"(p.19).

The view of reading as socioculturally transmitted literacy affects the assessment of reading. What it means to be literate, how this literacy is valued, used and displayed, will vary from culture to culture. The values implicit in the way reading is assessed may be culturally alien and therefore biased. The second implication of this recent view of reading as part of literacy is a tendency to downplay the psycholinguistic skill element in reading, and to emphasize the sociolinguistic aspects of literacy. While accepting that a view of reading as a skill is a narrow and possibly limited view of the nature of reading, it has not yet been worked through what an alternative view might mean in assessment terms.

\section{Process and Product Approaches to Reading}

Just as Alderson (2000) notes, it is commonplace in reading literature to distinguish between the process of reading and the product of reading, which is the result of that process. The process is what we usually mean by reading: the interaction between a reader and the text. During that process, many things happen. Not only are readers looking at print, decoding the marks on the page, deciding what the marks mean and how they relate to each other, readers are also thinking about what they are reading: what it means to them, how it relates to other things they have read, to things they know, and to what they expect to come next in the text. Evidently, many different things can be going on when a reader reads: the process is likely to be dynamic, variable, and different for the same reader on the same text at a different time or with different purpose in reading. And it is even more likely that the process will be different for different readers on different texts at different times and with different purposes. Thus, understanding the process of reading is important to the understanding of the nature of reading. But at the same time it is evidently a difficult thing to do. The process is normally silent, internal, and private.

Earlier research has focused on examining the eye movements of readers and insights have been gained from eye movement photography. However, watching what the eyes are doing may not tell us what the brain is doing, because "what the brain tells the eye is more important than what the eye tells the brain"(Smith, 1971). Asking the reader to read aloud is an alternative to eye movement photography as a means of externalizing the reading process, and one of the methods of investigating the reading-aloud process is miscue analysis (Goodman, 1969). Yet reading aloud is not the normal way in which people read, and the process of reading aloud is different from reading silently. Many other research methodologies, such as introspection and interview, have been used to investigate the reading process.

Inspecting the product of reading is an alternative approach to examining the process of reading. It is sometimes considered that, although different readers may engage in very different reading processes, the understandings they end up with will be similar. Thus, although there may be different ways of reaching a given understanding, what matters is not how a reader reaches that understanding, but what understanding a reader reaches. The problem of potentially infinite variation in reading processes is then reduced by a focus on what one has understood.

Earlier research into reading in the twentieth century used a product approach. Researchers typically designed tests of understanding of particular texts, administered the tests, and then inspected the relationship between the test results and variables of interest. Later, product approaches became unfashionable "as research efforts have concentrated on understanding the reading process, and as teachers of reading have endeavored to improve the way in which their students approach text (Alderson, 2000). Both a growing realization that processes of reading are more complex than originally assumed and the swing in research and teaching fashions, have led to revived interest in the product of reading.

According to Alderson (2000), there are at least two limitations to product approaches to reading, one is the 
variation in the product, the other is the method used to measure the product. It is obvious that what a reader understands from a text is affected by memory. Leaving aside the factor of memory, readers still may reach different understandings of the same text. This is because a text does not contain a meaning which is waiting to be discovered by a reader. Meaning is only realized in the interaction between the text and the reader, the text has "meaning potential" (Halliday, 1979; Widdowson, 1979). As is seen later, different readers have different backgrounds and experiences, and these will influence their understanding of a text, so the products of reading will certainly differ.

Given such differences in understanding, how can one determine which understanding is correct and which one is incorrect? Post-modernists would say that all products are possible and therefore are equally wrong or right. However, in reality, there must be some common-sense understanding or interpretation. But how to describe the criteria remains a particular issue, especially for test constructors.

The second limitation mentioned above is the method used to measure the product of understanding. Certain method (e.g. recall protocols) involves readers recalling what they have read without the text, so it is difficult to distinguish understanding from remembering. If readers are not familiar with testing approaches, then one risks the test method effect.

To summarize, it is possible to see reading as a process, or to examine the product of that process. Any theory of reading is likely to be affected by the emphasis that is placed on process or product. Product is easier to investigate than process, although it is not without problems.

\section{An Overview of Reading Models}

\subsection{Bottom-up Model}

A bottom-up reading model is a reading model that emphasizes the written or printed text, it says that reading is driven by a process that results in meaning (or, in other words, reading is driven by text) and that reading proceeds from part to whole.

To elaborate, Gough (1972) proposes a phonics-based or bottom-up model of the reading process which portrays processing in reading as proceeding in serial fashion, from letter to sound, to words, to meaning, in the progression suggested in the accompanying figure.

\section{Insert Figure 1 Here}

Stated in Gough's terms the reading system, from a bottom-up perspective, functions in sequences as follows. First, the graphemic information enters through the visual system and is transformed at the first level from a letter character to a sound, that is, from a graphemic representation to a phonemic representation. Second, the phonemic representation is converted, at level two, into a word. The meaning units or words then pass on to the third level and meaning is assimilated into the knowledge system. Input is thus transformed from low-level sensory information to meaning through a series of successively higher-level encodings, with information flow that is entirely bottom-up, no higher level processing having influence on any lower level processing. This process is also referred to as data-driven.

However, some researchers (e.g. Rumelhart, 1977) have already noticed the weaknesses of the bottom-up model in which processing is seen as proceeding only in one direction, so this implies that no higher level information ever modifies or changes lower level analysis. In some cases, readers are able to identify a word correctly only by employing higher level semantic and syntactic processing.

\subsection{Top-down Model}

A top-down reading model is a reading approach that emphasizes what the reader brings to the text, it contends that reading is driven by meaning and proceeds from whole to part. It is also known as concept-driven model.

To these theorists (e.g. Goodman, 1967; Smith, 1971), efficient reading doesn't result from the precise perception and identification of all the elements in a word, but from skills in selecting the fewest, most productive cues necessary. They contend that readers have a prior sense of what could be meaningful in the text, based upon their previous experiences and their knowledge about language. Readers are not, in their view, confined only to one source of information - the letters before their eyes, but have at their disposal two other important kinds of information which are available at the same time: semantic cues (meaning), and syntactic cues (grammatical or sentence sense). Thus, what readers bring to the text separately in terms of both their prior knowledge of the topic and their knowledge about the language assists them in predicting what the upcoming words will be. Readers sample the print, assign a tentative hypothesis about the identity of the upcoming word and use meaning to confirm their prediction. If meaning is constructed, readers resample the text and form a new hypothesis. Thus 
readers need to only briefly sample the marks on the page in order to confirm word identity.

In this model it is evident that the flow of information proceeds from the top downward so that the process of word identification is dependent upon meaning first. Thus the higher level processes embodied in past experiences and the reader's knowledge of the language pattern interact with and direct the flow of information, just as listeners may anticipate what the upcoming words of speakers might be. This view identifies reading as a kind of "psycholinguistic guessing game"(Goodman, 1967). A representation of the top-down process is depicted in the following figure.

Insert Figure 2 Here

The top-down model centers upon the assumption that good readers bypass the letter sound correspondence when they read because they read so quickly. That is, because good readers read at a faster speed, they do not depend upon the phonemic code. However, this view is also challenged. Recent evidence presented by Stanovich (1980) discredits this assumption. A lot of research suggests that instead of depending on meaning only, good readers may well markedly attend to graphic information, especially when they are uncertain about a word. Contrary to the view of the top-down theorists, good readers do rely on graphic information, which may be more efficient than endeavouring to predict words based only upon context and language structure. Moreover, the fact that good readers make better use of contextual clues than poor readers is not evidence that they actually do so in reading. Good readers use context only when orthographic and phonemic cues are minimal. Despite the view of top-down theorists then, it would appear that even as readers become more accomplished they still employ data-driven strategies to unlock words.

\subsection{Interactive Model}

Since neither the bottom-up nor top-down model of the reading process totally accounts for what occurs during the reading process, Rumelhart (1977) proposes an interactive model in which both letter features or data-driven sensory information and non-sensory information come together at one place. Using a computer analogy, Rumelhart labels this place a "message board". In this model, reading is not viewed simply as either a bottom-up or top-down process, but instead as a synthesizing of patterns, calling for the application or integration of all of the previously identified knowledge sources, as shown in the accompanying figure.

Insert Figure 3 Here

Here in the message board or pattern synthesizer as suggested in the accompanying diagram, orthographic knowledge, lexical knowledge, syntactic knowledge, and semantic knowledge come together simultaneously to facilitate word identification. It is theorized that the message center keeps a running list of hypotheses about the nature of the input string, scans the message board for the appearance of hypotheses relevant to its own sphere of knowledge, and then evaluates that hypothesis which is either confirmed or disconfirmed. Reading, according to Rumelhart, is thus neither a bottom-up nor top-down process, but a synthesis of the two.

Stanovich (1980) proposes an interactive-compensatory model, which adds a new feature to the interactive Rumelhart Model by suggesting that strength in one processing stage can compensate for weakness in another. According to Stanovich, problems in both the bottom-up and top-down models can be reduced with his model. That is, bottom-up models do not allow for higher-level processing strategies to influence lower-level processing, and top-down models do not account for the situation in which a reader has little knowledge of a text topic and, therefore, can not form predictions. In Stanovich's words, "Interactive models assume that a pattern is synthesized based on information provided simultaneously from several knowledge sources. The compensatory assumption states that a deficit in any knowledge source results in a heavier reliance on other knowledge sources, regardless of their level in the processing hierarchy"(1980, p. 63). Stanovich's theory explains the apparent anomaly found in many experiments in which poor readers sometimes show greater sensitivity to contextual constraints than do good readers. Poor readers may be thus using strong syntactic or semantic knowledge to compensate for less knowledge of orthography or lexicon.

\section{Reading Research in China}

In China, great progress has been made in the studying of reading. Zeng $(1992,1999)$ writes a series of books on reading, his works are seen as the latest development of the reading research in China. Zeng contends that reading, as a complete discipline, should cover the principles of reading, reading techniques or skills, and reading training, while the principles of reading are at the core of reading studies.

Zeng considers reading as a philosophical system which is composed of reading object, reading subject and reading noumenon. The reading object (reading material, reading environment, reading time, and reading tool) studies show that reading is an open system in which readers, reading materials and reading environment interact 
with one another, it occurs in a certain time chain and can only achieve the information exchange between the reader and reading materials with a certain tool. The reading subject (reading philosophy, reading psychology, reading intelligence and reading accomplishment) studies show that reading is not only a unity of philosophical activity and psychological activity, but also a transformation of the information in the reading materials and readers' accomplishments. The reading noumenon is the connection and unity of reading object and reading subject, it relates to reading nature, the values of reading, reading objective, reading process, reading pathology, and aesthetics in reading.

References

Alderson, J. C. (2000). Assessing reading. Cambridge: Cambridge University Press.

Barnett, M. A. (1989). More than meets the eye Foreign language reading: Theory and practice. New Jersey: Prentice-Hall.

Bernhardt, E. B. (1991). Reading development in a second language: Theoretical, empirical, and classroom perspectives. Norwood, NJ: Ablex.

Goodman, K. S. (1967). Reading: A psycholinguistic guessing game. Journal of the Reading Specialist, 6, 126-135.

Goodman, K. S. (1969). Analysis of oral reading miscues: Applied psycholinguistics. Reading Research Quarterly, 5, 9-30.

Gough, P. B. (1972). One second of reading. In J. F. Kavanagh, \& I. G. Mattingly (Eds.), Language by ear and by eye. Cambridge, MA: MIT Press.

Halliday, M. A. K. (1979). Language as social semiotic. London: Edward Arnold.

Rayner, K., \& Pollatsek, A. (1989). The psychology of reading. Englewood Cliffs, NJ: Prentice Hall.

Rumelhart, D. E. (1977). Toward an interactive model of reading. In S. Dornic (Ed.), Attention and performance VI. Hillsdale, NJ: Laurence Erlbaum Associates.

Smith, F. (1971). Understanding reading. New York: Holt, Rinehart and Winston.

Stanovich, K. E. (1980). Toward an interactive compensatory model of individual differences in the development of reading fluency. Reading Research Quarterly, 16, 32-71.

Urquhart, A. H., \& Weir, C. J. (1998). Reading in a second language: Process, product and practice. London and New York: Longman.

Wallace, C. (1992). Reading. Oxford: Oxford University Press.

Widdowson, H. G. (1979). Explorations in applied linguistics. Oxford: Oxford University Press.

Zeng Xiangqin.(1992).The Principles of Reading. Zhengzhou: Henan Educational Press

Zeng Xiangqin.(1999).A New Study of Reading. Beijing: Yuwen Press

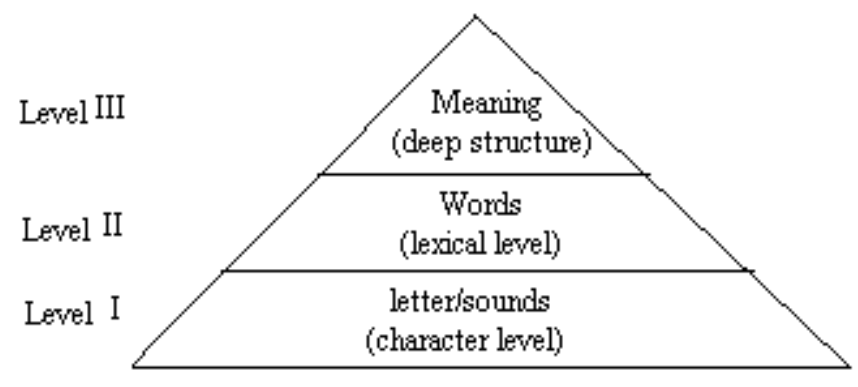

Figure 1. Data-driven or Bottom-up Model 


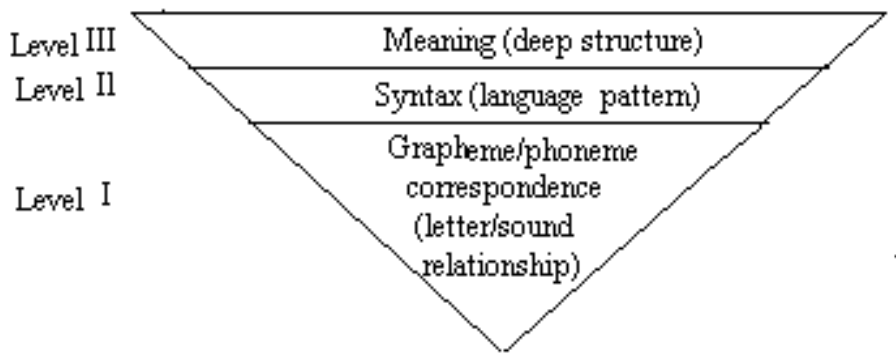

Figure 2. Hypothesis Test or Top-down Model

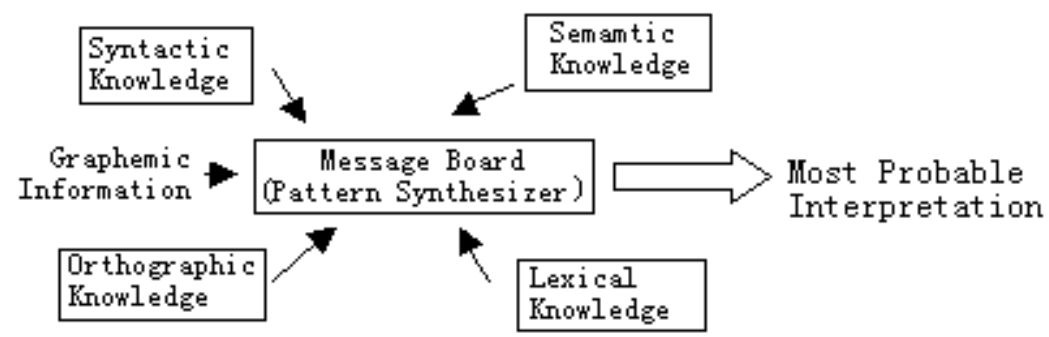

Figure 3. Interactive Model (adapted from Barnett, 1989, p. 24) 